Mots. Les langages du politique

\title{
Communication publique et violence intrafamiliale
} au Chili

De la femme victime à la femme empoderada

Communication campaigns and domestic violence in Chile. After victim women, the empowered women

Comunicación pública y violencia intrafamiliar en Chile. De la mujer víctima a la mujer empoderada

\section{Myriam Hernández Orellana}

\section{(2penEdition}

\section{Journals}

Édition électronique

URL : https://journals.openedition.org/mots/22114

DOI : $10.4000 /$ mots. 22114

ISSN : 1960-6001

\section{Éditeur}

ENS Éditions

Édition imprimée

Date de publication : 30 novembre 2015

Pagination : 67-82

ISBN : 978-2-84788-776-1

ISSN : 0243-6450

Référence électronique

Myriam Hernández Orellana, «Communication publique et violence intrafamiliale au Chili », Mots. Les langages du politique [En ligne], 109 | 2015, mis en ligne le 30 novembre 2017, consulté le 23 avril 2022 URL : http://journals.openedition.org/mots/22114 ; DOI : https://doi.org/10.4000/mots.22114 


\section{Communication publique et violence intrafamiliale au Chili. De la femme victime à la femme empoderada}

Au Chili'i la politique publique pour prévenir les violences contre les femmes au sein du couple a pris de l'ampleur pendant le premier gouvernement de Michelle Bachelet ${ }^{2}$ (2006-2010). Différents instruments de l'action publique ${ }^{3}$ ont été mis en œuvre, tandis que d'autres, notamment l'instrument informatif et communicationnel, ont vu croître leur importance : d'une part, le budget annuel dédié aux campagnes de prévention sur le sujet a augmenté; d'autre part, le Service national de la femme (Servicio Nacional de la Mujer, Sernam) ${ }^{4}$ a développé une véritable offensive communicationnelle afin de mettre la violence au sein du couple à l'agenda médiatique et public. Cette offensive a consisté à faire connaître le concept de "fémicide " (Hernández Orellana, 2012) afin de rendre visible la violence intrafamiliale ; elle a consisté aussi à réaliser chaque année des campagnes de communication pour prévenir celle-ci. L'importance acquise par la communication de l'action publique au cours des années Bachelet relève de ce que certains auteurs appellent la « dimension communicationnelle de l'action publique» (Ollivier-Yaniv, 2013).

Au Chili, l'une des premières actions du Sernam fut de légiférer sur les violences contre les femmes, notamment au sein du couple. Ainsi, en 1994, fut promulguée la loi $n^{\circ} 19.325$ sur la violence intrafamiliale (Ley de violencia intrafamiliar) ${ }^{5}$, laquelle ne distingue pas le sexe des victimes et des agresseurs car elle s'attaque aux violences produites dans la famille en général. Ainsi le «bien » juridique que l'État entend protéger est la famille car elle est garante du maintien de la société. Dans ce schéma d'interprétation, les femmes ont

1. Au Chili, l'action publique contre ces violences s'intègre dans la lutte pour l'équité entre les femmes et les hommes (voir Sernam, 2007).

2. Michelle Bachelet a été réélue présidente le 15 décembre 2013, pour la période 2014-2018.

3. Par instrument de l'action publique (IAP), nous entendons « un dispositif à la fois technique et social qui organise les rapports sociaux spécifiques entre la puissance publique et ses destinataires en fonction des représentations et des significations dont il est porteur » (Lascoumes, Le Galès, 2004, p. 13).

4. Créé en 1991, le Sernam est l'organisme gouvernemental chargé des politiques d'égalité entre les femmes et les hommes (loi n $\left.{ }^{0} 19.023\right)$.

5. Le texte a été promulgué le 27 août 1994. 
été ignorées en tant que sujets de droit (Araujo et al., 2000). Les chercheuses féministes chiliennes ont critiqué la loi, car celle-ci assimile les violences exercées contre les femmes à n'importe quelle violence au sein d'une famille, et nie leur caractère genré. Le 7 octobre 2005 , la loi n²0.066 a remplacé le texte de 1994 afin de durcir les mesures contre les agresseurs. Mais le nom «violence intrafamiliale ${ }^{6}$ a été conservé. À la différence de ce qui a été accordé sur le plan international 7 , la loi chilienne ne reconnaît pas que les femmes, en tant que telles, peuvent être victimes de violences parce qu'elles sont des femmes.

L'utilisation par Madame Bachelet du genre en tant que ressource en politique (Marques-Pereira, 2011) a abouti à l'établissement de l'Agenda de genre (Agenda de Género), mis en place en 2007. Ce document établit les priorités et engagements pris par le gouvernement afin d'avancer vers l'équité entre les sexes. L'Agenda de genre ne constitue pas une rupture vis-à-vis de l'action déjà menée par le Sernam depuis sa création, mais plutôt un recadrage significatif de ses objectifs. Il fait «pencher la balance un peu plus du côté de l'individuation des femmes que du familialisme » (Forstenzer, 2011), lequel avait jusqu'alors cadré la politique publique menée par l'institution. Bien que le gouvernement Bachelet n'ait pas changé le nom de la loi, le recadrage de la politique publique explicité dans l'Agenda de Genre permet de reconnaître les femmes en tant que victimes, ainsi que l'importance du rôle des sexes assignés par la société et qui construisent l’identité des individus.

Tout ce qui précède nous amène à proposer l'hypothèse suivante : en essayant de faire évoluer les rapports du pouvoir à l'intérieur du couple, les campagnes de communication de prévention réalisées pendant le premier gouvernement Bachelet et destinées au grand public ont construit symboliquement l'identité des hommes (agresseurs) et celle des femmes (victimes). Notre article s'inscrit dans l'approche qui considère que, sur des sujets d'intérêt général, la communication publique vise à réguler les représentations et les comportements des citoyens (Ollivier-Yaniv, 2006).

En questionnant les représentations des femmes victimes et des hommes agresseurs, ce texte s’intéresse à l’identité de genre ${ }^{8}$, compris comme le «fait

6. L'article 5 de la loi définit la violence intrafamiliale comme « toute maltraitance qui affecte la vie ou l’intégrité physique ou psychique de celui qui a ou qui a eu la qualité de conjoint de l'offenseur, ou une relation de concubinage avec lui ; ou qui est un parent par consanguinité ou affinité dans toute la lignée directe ou indirecte jusqu'au troisième degré de l'offenseur, ou de son conjoint ou de son concubin actuel». (Ma traduction)

7. En 1994, les pays membres de l’Organisation des États américains ont adopté la «Convention interaméricaine pour prévenir, sanctionner et éradiquer la violence contre la femme, "Convention de Belém do Pará” " (ma traduction). Le texte inclut les violences commises dans les sphères privée et publique, réalisées par des proches de la victime ou par des personnes inconnues de celle-ci, ainsi que les violences tolérées ou perpétrées par l’État.

8. Nous comprenons le genre comme « un système de bi-catégorisation hiérarchisé entre les sexes (hommes/femmes) et entre les valeurs et représentations qui leur sont associées (masculin/ féminin)»(Bereni etal., 2008). 
pour un être humain de se penser et de se sentir comme appartenant à un sexe » (Bereni et al., 2008). Cette identité de genre est développée par les enfants lors du processus de socialisation, pendant lequel la famille et l'école ont une influence prépondérante. Les rôles de sexes, différents pour les garçons et les filles, constituent la première dimension de leur socialisation de genre. Comme l'explique Erving Goffman, les rôles sociaux des hommes et des femmes sont généralement différenciés dans la famille, ce qui attribue accidentellement aux femmes une place et un pouvoir moindres : exclusion de la guerre et de la chasse, restriction de l'usage qu'elles peuvent faire de l'espace public... Plus que les hommes, elles "voient leur vie centrée sur les tâches domestiques» (Goffman, 2002).

Afin d'éclairer les représentations des femmes victimes et des hommes agresseurs produites par la communication du gouvernement Bachelet, et dans le but d'identifier les violences représentées, nous avons réalisé une analyse de contenu et sémiologique de quatre campagnes de communication de prévention contre la violence intrafamiliale mises en œuvre pendant le premier gouvernement de Mme Bachelet. Au total, notre corpus est constitué de dix affiches, dix spots radiophoniques et trois spots télévisés, ce qui correspond à l'ensemble des dispositifs de communication mis en place par le gouvernement entre 2006 et 2009 . Pour l'analyser, nous nous appuyons sur la sémiologie des indices, laquelle s'intéresse au processus de conventionnalisation de formes signifiantes (Houdebine, 1994). L'analyse se réalise à travers trois étapes : systémique, explicative et interprétative. Les deux premières étapes constituent l'analyse systémique immanente qui a pour but d'éviter les interprétations trop rapides. La phase explicative cherche à dégager le mode de fonctionnement de ces indices. La phase interprétative «révèle les insu (sic) idéologiques ou stéréotypes socio-culturels utilisés, et dans ses analyses communicationnelles elle permet d'évaluer les messages émis relativement aux intentions des émetteurs ou de les ajuster eu égard aux réceptions » (Houdebine, 2010). La sémiologie des indices propose un cadre d'analyse souple, car les strates à partir desquelles est étudié le corpus sont définies par le chercheur en fonction de ces observables. Ainsi, Anne-Marie Houdebine propose trois strates (scénique, iconique, linguistique), tandis que d'autres auteurs en ont construit de différentes. Dans son article consacré à la publicité pour les parfums, Jean-Claude Soulages a étudié les différentes modalités des mises en scène et la figuration des identités sexuelles. Il a établi ses analyses à partir d'une strate "plastique», de celle «des éléments figurés» et de celle qui concerne les modes d'organisation discursive (dite «narrative»). Nous avons repris ces trois strates tout en les adaptant à notre corpus ${ }^{9}$. Nous avons aussi

9. La strate plastique comprend : le cadrage (horizontal / vertical); l'arrière plan (net / flou); les formes (aiguës et rigides/douces) ; le canal (dessins/photos) ; l'emploi de couleurs (chaudes / froides) ou utilisation du noir et blanc; la lumière (directe et dure / naturelle et douce); le plan 
utilisé la "strate scénique» pour la description physique des personnes et des objets, afin de déterminer le niveau social ${ }^{10}$, l'âge ${ }^{11}$, etc., des personnes représentées dans ces campagnes de prévention. De plus, nous avons décidé d'ajouter les strates relatives aux mouvements d'humeur ${ }^{12}$, à la prosodie ${ }^{13}$ et aux effets de son ${ }^{14}$, afin de tenir compte aux mieux des vidéos et des spots radiophoniques de notre corpus.

Les campagnes de communication pour prévenir les violences intrafamiliales étudiées constituent le discours institutionnel du gouvernement Bachelet sur le phénomène des violences intrafamiliales, ce qui nous a conduit à solliciter des corpus institutionnels emboîtés ${ }^{15}$ (Oger, Ollivier-Yaniv, 2003), éclairant les conditions de réalisation des campagnes médiatiques en tant que telles. Notre démarche méthodologique cherche à mettre en lumière les identités des femmes victimes et des hommes agresseurs produites par la communication du gouvernement Bachelet (2006-2010), sans nous intéresser à la réception que ces représentations peuvent susciter dans le grand public.

Dans une première partie, nous étudierons l'identité de genre au Chili dans les couples où se produisent des violences intrafamiliales, telle qu'elle a été décrite par des chercheuses féministes, car ces définitions font partie du référentiel de la politique publique ${ }^{16}$ qui a cadré celle destinée à prévenir ce phénomène. Dans une deuxième partie, nous analyserons les identités des acteurs construites par ces campagnes de communication.

des images (premier plan / arrière plan, etc.). La strate d'éléments figuratifs comprend : l'univers déréalisé / réel ; le point de vue (hauteur de l'œil / plongée / contreplongée) ; le regard (autocentré / vers l'agresseur / autre) ; les gestes : bouche et yeux ouverts / fermés, sourcils (froncés / vers le haut), positions des mains, des jambes, de la tête, des épaules; les pleurs et le rire) ; les positions des acteurs dans le cadrage du visuel / spot TV; les traits du visage. La strate narrative comprend : la contextualisation de la violence; la signification des couleurs; la signification des messages; le type de regard ; l'attitude des acteurs; les stéréotypes véhiculés.

10. Le fait de porter ou non des bijoux, de quel type (en or, en perles, de fantaisie, etc.) ; le type de vêtements; la coupe de cheveux; le type de décoration des appartements / maisons (tableaux, affiches, etc.); le revêtement de sol (carrelage, lino, tapis); le type des fenêtres; le type des meubles...

11. Nous avons identifié l'âge des personnes dans les campagnes par la couleur et la coupe de cheveux, le fait d'avoir des enfants en bas âge, les rides sur le visage, le ton de la voix, la façon de s'habiller, etc.

12. Éléments tels que : grimacer, se prendre la tête dans les mains, approcher les mains de la poitrine, taper du poing sur la table, etc.

13. Éléments tels que : voix très basse, voix forte, cris, etc.

14. Éléments tels que : bruits de pleurs, bruits de verre cassé, bruits de rue, claquements / ouvertures de portes, bruits de serrure, etc.

15. Tels que les documents internes de préparation des campagnes, les dossiers de presse pour les présentations des campagnes, les communiqués de presse sur les campagnes, voire des entretiens avec la ministre du Sernam de l'époque, Laura Albornoz.

16. Nous utilisons ici la notion de référentiel d'une politique publique de Pierre Muller : «Il s'agit à la fois d'un processus cognitif permettant de comprendre le réel en limitant sa complexité et d’un processus prescriptif permettant d'agir sur le réel » (1990, p. 63). 


\section{Violence intrafamiliale et modèle patriarcal}

C'est à Nieves Rico que l'on doit l'un des premiers travaux sur la violence au sein du couple en Amérique latine. L'auteure a associé ce phénomène au système patriarcal, dans lequel l'homme exerce le pouvoir à l'intérieur de la famille et dans la société. La violence est «l'un des instruments qui permettent de perpétuer la suprématie masculine » (Rico, 1992). Plusieurs auteures s'accordent sur l'idée que dans de telles sociétés, les hommes sont encouragés à être agressifs et violents tandis que les femmes doivent se montrer soumises et dépendantes. Ainsi, lors du processus de socialisation des enfants, on attend que les garçons manifestent leur agressivité (en se battant, par exemple). En revanche, les familles apprennent aux filles «à soigner, à éduquer, à être responsable[s] du bien-être des autres, à partager le pouvoir, à résoudre les conflits sans utiliser la violence, à construire des structures égalitaires» (Larraín, Rodríguez, 1993). Un élément particulier à l'Amérique Latine est le marianismo ${ }^{17}$, qui renforce l'identité de genre.

Dans ce schéma, on attend que la femme latino-américaine imite le modèle mariano [marial] de perfection et supériorité spirituelle, tandis que la valeur de sa personne se base sur la maternité. De plus, le rapport avec l'homme est marqué par la soumission et l'obéissance. ${ }^{18}$

Au Chili, la recherche sur la violence contre les femmes au sein du couple s'est développée à partir des sphères institutionnelle et militante ${ }^{19}$, lesquelles se croisent de façon permanente ${ }^{20}$. C'est à partir des travaux réalisés par des chercheuses féministes et, par la suite, de ceux développés par le Sernam, que cet organisme gouvernemental a construit les instruments permettant d'aborder ce problème. La recherche sur la violence contre les femmes a été fortement encouragée par l'organisme lui même, à travers la création d'une unité de recherche.

Au Chili, vers la fin des années quatre-vingt et au début des années quatrevingt-dix, très peu d'études concernaient ce phénomène; de plus, elles portaient toutes sur des milieux défavorisés. Partant de ces recherches, Soledad Larraín

17. D'après les auteures, le marianismo est compris comme une version du culte de la Vierge Marie, qui incarne simultanément l'idéal de maternité et de chasteté.

18. Texte original (Larraín, Rodríguez, 1993) : «Bajo este esquema se espera que la mujer latinoamericana imite el modelo mariano de perfección y superioridad espiritual, mientras que la valoración de su persona se fundamenta en la maternidad. La relación con el hombre está marcada, adicionalmente, por la sumisión y la obediencia. » (Ma traduction)

19. Plusieurs associations féministes, devenues des ONG au cours des années quatre-vingt-dix, ont développé la recherche sur l'égalité femmes/hommes grâce à des fonds internationaux et à la réalisation des projets scientifiques commandés par le Sernam.

20. S. Larraín, première sous-directrice de l'institution, était une chercheuse féministe reconnue. Deux ans après avoir démissionné du Sernam, elle a publié en 1994 le premier livre s'attaquant à ce problème au Chili : Violencia puertas adentro: la mujer golpeada (Violence dans la maison: la femme battue; ma traduction). 
et Teresa Rodríguez ont publié en 1993 l'un des premiers articles sur la violence familiale au Chili. Elles y inscrivent la violence contre la femme dans « un système de pouvoir basé sur la supériorité masculine, où se déterminent les fonctions sociales des hommes et des femmes sur la base de leur sexe » (Larraín, Rodríguez, 1993). Ce trait est caractéristique de la famille patriarcale, dont le modèle hégémonique véhicule une idéologie visant à faire de la discrimination un principe fondateur des relations entre hommes et femmes. L'article définit ce type de violences comme les actes - y compris verbaux, tels que les menaces qui ont «pour objectif de contraindre une femme à adopter un comportement déterminé » (Larraín, Rodríguez, 1993). Certaines situations dont l'article indique qu'elles déclenchent les violences conjugales (la jalousie, le refus d'avoir des relations sexuelles, etc.) manifestent les rapports inégaux entre les sexes.

Les auteures proposent une sorte de portrait psychologique des hommes agresseurs et des femmes victimes à partir des études sur le processus de socialisation dans des familles autoritaires. L'agresseur type a une faible estime de lui-même, il témoigne également d'un manque de confiance en soi, ce qu'il essaye de compenser en adoptant des attitudes autoritaires au foyer. Il communique mal, est dans l'incapacité de tolérer et de résoudre un conflit en utilisant d'autres moyens que la violence. Enfin, il n'est pas conscient de celle-ci et tout se passe comme si la responsabilité de ses actes incombait à son entourage.

Selon les auteures, la plupart des femmes concernées répondent aux attaques en se protégeant ou en pleurant, et une minorité seulement demande de l'aide. Lors d'une agression, elles expriment des sentiments de rage et d'impuissance. Elles quittent leur travail, leurs proches, s'isolent, ce qui augmente leur dépendance envers leur agresseur. De plus, elles se sentent responsables de ces agressions répétées, qui deviennent à leurs yeux des souffrances justifiées prouvant une forme d'échec dans leur comportement.

Dans les paragraphes qui suivent, nous analyserons comment l'État a lutté contre le modèle de la famille patriarcale à travers les campagnes de prévention des violences intrafamiliales.

\section{La construction symbolique des "hommes-agresseurs » et des «femmes-victimes »}

Les différents documents produits par le service de communication du Sernam autour des campagnes réalisées entre 2006 et 2008 mettent en évidence que leur objectif est de sensibiliser les Chiliens aux violences envers les femmes ${ }^{21}$. Ainsi, la campagne de 2006 cherche à sensibiliser les hommes agresseurs pour

21. Les catégories des violences (physique, psychologique et verbale, sexuelle) analysées ici sont inspirées de la classification de Maryse Jaspard, dans l'enquête ENVEFF (Jaspard, 2011). 
Tableau 1. Campagnes par types de dispositifs communicationnels utilisés et types de violences représentées

\begin{tabular}{|c|c|c|c|c|c|}
\hline $\begin{array}{l}\text { Campagne } \\
\text { (année) }\end{array}$ & $\begin{array}{l}\text { Type de dispositif } \\
\text { communicationnel }\end{array}$ & $\begin{array}{l}\text { Violence } \\
\text { physique }\end{array}$ & $\begin{array}{l}\text { Violence } \\
\text { psycholo- } \\
\text { gique (et } \\
\text { verbale) }\end{array}$ & $\begin{array}{l}\text { Violence } \\
\text { sexuelle }\end{array}$ & $\begin{array}{c}\text { Diverses } \\
\text { violences } \\
\text { dans une } \\
\text { seule affiche } \\
\text { ou spot }\end{array}$ \\
\hline \multirow[t]{2}{*}{$2006^{*}$} & affiches (3) & $1 / 3$ & $1 / 3$ & $\begin{array}{c}1 / 3 \\
\text { (implicite) }\end{array}$ & $\begin{array}{l}1 / 3 \text { phys. } \\
\text { et psych. }\end{array}$ \\
\hline & spot radio (3) & $1 / 3$ & $1 / 3$ & $0 / 3$ & $\begin{array}{l}1 / 3 \text { phys. } \\
\text { et psych. }\end{array}$ \\
\hline \multirow[t]{3}{*}{2007} & affiches (3) & $3 / 3$ & $0 / 3$ & $0 / 3$ & $0 / 3$ \\
\hline & spot radio (3) & $2 / 3$ & $1 / 3$ & $0 / 3$ & $0 / 3$ \\
\hline & spot tv (1) & $1 / 1$ & $1 / 1$ & $0 / 1$ & $\begin{array}{l}1 / 1 \text { phys. } \\
\text { et psych. }\end{array}$ \\
\hline \multirow[t]{3}{*}{2008} & affiches (1) & $1 / 1$ & $0 / 1$ & $0 / 1$ & $\begin{array}{l}1 / 1 \text { phys. } \\
\text { et psych. }\end{array}$ \\
\hline & spot radio (3) & $1 / 3$ & $2 / 3$ & $0 / 3$ & $\begin{array}{l}1 / 3 \text { phys. } \\
\text { et psych. }\end{array}$ \\
\hline & spot tv (1) & $1 / 1$ & $1 / 1$ & $0 / 1$ & $\begin{array}{l}\text { / } 3 \text { phys. } \\
\text { et psych. }\end{array}$ \\
\hline \multirow[t]{3}{*}{2009} & affiches (3) & \multirow{3}{*}{$\begin{array}{c}\text { sans } \\
\text { violence }\end{array}$} & \multirow{3}{*}{$\begin{array}{c}\text { sans } \\
\text { violence }\end{array}$} & \multirow{3}{*}{$\begin{array}{c}\text { sans } \\
\text { violence }\end{array}$} & \multirow{3}{*}{$\begin{array}{c}\text { sans } \\
\text { violence }\end{array}$} \\
\hline & spot radio (1) & & & & \\
\hline & spot tv (1) & & & & \\
\hline
\end{tabular}

*La campagne de communication de l'année 2006 n'a pas utilisé de spot de télévision.

**Entre parenthèses, le nombre total des dispositifs utilisés pour chaque campagne.

Source : Hernández Orellana, 2014.

leur faire comprendre que la violence qu'ils exercent est douloureuse pour leurs victimes. Celle de 2007 s'adresse à la société dans son ensemble pour que les témoins des violences les dénoncent, tandis que la campagne de 2008 s'attaque aux stéréotypes qui produisent ces violences, afin de provoquer un changement culturel à travers de nouveaux rapports entre les sexes.

Le tableau ci-dessus montre que pendant la période du premier gouvernement Bachelet, les campagnes de communication sont caractérisées par une surreprésentation de la violence physique. La plupart des visuels, spots radio et spots TV des campagnes réalisées entre 2006 et 2008 la mettent en scène. Les spots radiophoniques ${ }^{22}$ insistent davantage sur la violence psychologique; cependant, certains effets sonores comme un bruit de verre cassé ou un claquement de porte y ont été utilisés pour suggérer la violence physique.

22. Dans l'article, nous utilisons A pour les affiches, SR pour les spots radiophoniques, STV pour les spots TV, et CE pour les captures d'écran des STV). 
Il est important de noter que la violence sexuelle au sein du couple n'est évoquée que dans une seule affiche de la campagne de 2006. Les femmes victimes de violence physique sont les plus représentées dans le discours institutionnel au cours de la période étudiée (Hernández Orellana, Kunert, 2013). Cependant, plusieurs études du Sernam réalisées entre 1992 et 2005 dans différentes régions du pays, ainsi que la première enquête nationale sur le phénomène (2008), démontrent que c'est la violence psychologique qui provoque le plus grand nombre de victimes. D’ailleurs, le cahier des charges de la campagne de 2008 insiste sur la nécessité de s’éloigner des représentations de la violence extrême : toutes les femmes, en effet, ne s'identifient pas dans ce cas à la victime et la conséquence en est que nombre d'entre elles, à tort, ne se sentent pas visées au point de porter plainte (Comunicaciones Sernam, 2008). Ce document préconise donc la représentation des comportements quotidiens constitutifs d'une forme de violence qui n'est pas forcément identifiée comme telle.

\section{Portrait de l'homme en agresseur}

Les spots radiophoniques véhiculent un portrait psychologique de l'homme agresseur qui correspond à celui qu'ont élaboré Larraín et Rodríguez, cités plus haut. L'homme agresseur est jaloux; il est en manque de sécurité car il soupçonne sa femme de le tromper: "Je ne veux pas que tu t'habilles comme ça, autant de maquillage... qui veux-tu draguer? C'est sûr que tu sors avec un autre $\mathrm{mec}$ » (SR 2007); il rabaisse sa femme : «Tu es moche», "Tu ne sais rien» (SR 2006) et cherche à contrôler le comportement de celle-ci : "Habille-toi décemment. Allez, fais ce que je dis. Va te changer» (SR 2007), «Je n'aime pas que tu sortes. Ta place est ici, avec moi » (SR 2008).

Mais les campagnes montrent d'autres caractéristiques de l'homme agresseur qui n'ont pas été signalées par Larraín et Rodríguez : l'autoritarisme et la manipulation. La première est présente dans les phrases exhortatives ou impératives décrites plus haut, ainsi que dans des phrases interrogatives telles que «Tu crois que tu es libre de faire ce que tu veux? Ça va pas, non?» (SR 2007), ou dans certains énoncés comme : «Attention à ce que tu vas dire », «Ici, c'est moi qui commande» (SR 2006). La deuxième, qui constitue un élément périphérique ${ }^{23}$, apparaît à travers la mise en scène d'un chantage émotionnel : «Si tu me quittes, je te jure que je me tue » (SR 2008). Ces caractéristiques sont utilisées aussi dans les affiches et les spots télévisés réalisés entre 2006 et 2008.

Les supports dont l'image est l'élément principal représentent l'homme agresseur dans l'exercice d'une violence physique. On le voit à l'expression

23. Selon Anne-Marie Houdebine, les «éléments périphériques » sont ceux dont l'usage reste très minoritaire. 


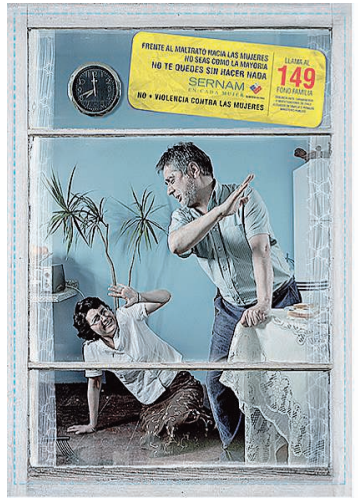

A 1

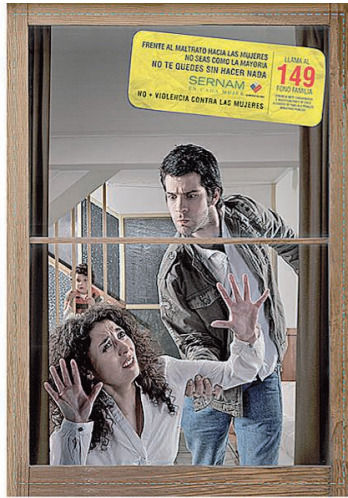

$\mathrm{A} 2$

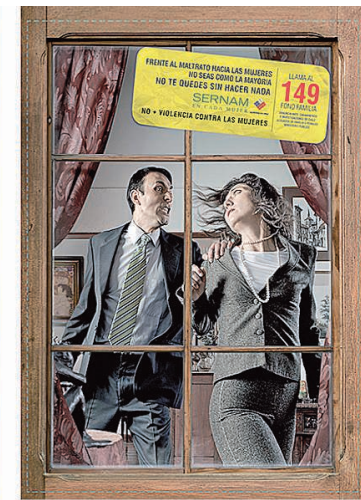

A3

Affiches de la campagne 2007

des visages et aux positions des mains des hommes dans les affiches reproduites ci-dessus ${ }^{24}$.

En A1, l'homme, placé au centre de l'affiche en position dominante et dynamique, esquisse du bras un geste qui révèle l'imminence d'une agression physique. Censé appartenir à la classe populaire, il s'apprête à frapper une femme qui se trouve à terre, sans défense. Tandis que l'agresseur qui représente le milieu aisé (A3) essaye de prendre par l'épaule une femme qui est à sa hauteur, et de la retournervers lui. On observe que les rapports de domination de l'homme envers la femme sont plus visibles dans l'affiche qui met en scène les violences au sein la classe populaire, entre partenaires plus âgés (A1), que dans l'affiche où sont représentés les couples de milieu aisé (A3) ou plus jeunes $(A 2)$. Un élément périphérique de notre analyse se trouve donc être la représentation des modalités de ces violences selon les couches sociales, réalisée uniquement par les affiches de 2007.

\section{Les femmes-victimes «construites» par les campagnes}

Entre 2006 et 2008 , les campagnes de communication du Sernam ont montré des femmes soumises : face à l'agression, elles semblent dans l'incapacité de réagir autrement qu'en pleurant ou en se protégeant de leurs mains. Dans aucune de ces campagnes, les femmes ne se défendent. Ces représentations sont paradoxales au regard de l'étude réalisée par le Sernam en 2002 auprès de femmes âgées de dix-huit à quarante-neuf ans, en couple dans la région de

24. Le slogan de la campagne 2007 est : «Ne reste pas sans rien faire» («No te quedes sin hacer nada »). Ma traduction. 
Metropolitana et dans celle d'Araucanía : celle-ci indique qu'une proportion non négligeable des femmes victimes de violences physiques - plus de la moitié de la première région et plus des deux tiers de la deuxième - se sont, dans ces circonstances, défendues en frappant leur agresseur (voir Sernam, 2009).

Malgré ce constat, la première campagne réalisée par le gouvernement Bachelet en 2006 représente une femme incapable d'agir, qu'il s'agisse de s'éloigner ou de se défendre. Dans les spots radiophoniques, les victimes racontent leur désespoir sur un ton de voix monocorde et passif : "Il m’a giflée et m’a balancé des objets dessus. Il m’a poussée et m’a coincée. Il m'a frappée du poing. Il m’a donné des coups de pied et il m'a traînée par terre. Il a voulu m'étrangler ». Le véritable protagoniste de l'action n'est pas la femme qui raconte ce qui lui est arrivé, mais l'agresseur, qui n'est pas identifié (mari, compagnon, etc.) par la victime dans son récit. C'est cette représentation de la victime qu'utilisent les affiches ci-dessous, censées s'adresser aux hommes agresseurs ${ }^{25}$ :

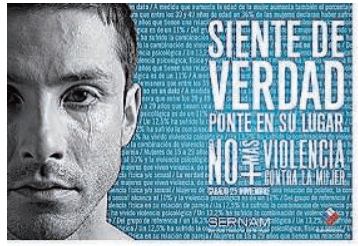

A4

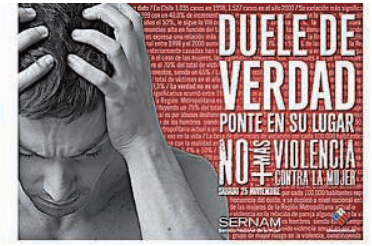

A5

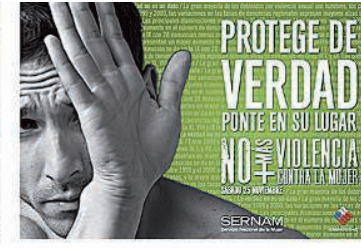

A6

Affiches campagne 2006

Nous l'avons expliqué dans d'autres publications (Hernández Orellana, Kunert, 2013) : l'inversion des rôles ici présente (l'homme prend la place de la femme victime), renforcée par le slogan «Mets-toi à sa place» ainsi que par les trois messages secondaires «Ressens pour de vrai » (A4), «Ça fait mal pour de vrai » $\left(A_{5}\right)$ et "Protège pour de vrai » $(A 6)$, ne fait que parodier les rapports de genre sans questionner les stéréotypes véhiculés. Dans ce cas, on peut se demander si, à travers la reproduction de ces stéréotypes, le discours institutionnel ne construit pas des identités de femmes victimes et d'hommes agresseurs.

Dans deux des affiches de la campagne de 2007 (A1 et A2), les femmes sont en position d'infériorité vis-à-vis de leur agresseur. Toutefois, nous observons quelques différences dans la représentation des victimes. Bien que la femme de l'affiche A2 soit à terre, elle appuie ses mains sur la fenêtre, dans un geste suppliant qui semble demander de l'aide à ceux qui se trouvent de l'autre côté, autrement dit au public de la campagne, éventuel témoin de telles violences. Pour sa part, la femme victime de l’affiche censée représenter le milieu

25. Le slogan de la campagne 2006 est : «Mets-toi à sa place» («Pónte en su lugar»). Ma traduction. 
aisé $\left(A_{3}\right)$ est debout et se trouve à la même hauteur que l'homme. Elle essaye de s'échapper, ce qui est signifié par le mouvement de sa chevelure ainsi que par la position avancée de sa jambe droite. Au contraire, dans l'affiche A1, on observe que la femme - plus âgée et de milieu plus modeste que dans A2 et A3 - semble démunie face à son agresseur : elle ne peut demander de l'aide car elle se trouve loin de la fenêtre, et comme elle est à terre et tente de se protéger, elle n'a pas la possibilité de se défendre ni de s'enfuir. Ainsi, dans l'affiche, la position de cette femme vis-à-vis de celle de son agresseur signifie une plus grande infériorité physique.

\section{La femme empoderada, une nouvelle identité pour les Chiliennes?}

Le marianismo a fait que la maternité occupe une place importante dans l'identité des femmes en Amérique Latine. Et pourtant, dans notre corpus, la présence des enfants demeure marginale. La campagne de 2007 est la seule à avoir mis en scène les enfants du couple, témoins des violences malgré eux ( $A_{2}$ et SR4). De plus, les enfants ne sont pas utilisés pour contraindre symboliquement la femme, mère et victime, de s'éloigner de l'homme, père et agresseur (ce qui aurait pour objectif d'empêcher que les enfants imitent le comportement violent de leur père). Autrement dit, dans ces campagnes de prévention, l'absence du rôle de mère dans la représentation des femmes victimes rompt avec l'identité des femmes associée à la maternité.

Mais la communication destinée à prévenir les violences intrafamiliales va progressivement mettre en scène un nouveau comportement des femmes victimes, comme le montre le spot télévisé de 2008 intitulé «Rien ne justifie les violences contre les femmes », dont on trouvera page suivante quelques captures d'écran.

Ce spot nous montre le moment où la femme prend conscience (CE4) qu'elle seule est en mesure de changer le rapport installé dans la relation de couple. Ici, l'agresseur s'excuse pour son comportement violent, pendant que la femme l'écoute en silence, tête baissée (CE1). Puis la femme victime est harcelée par sa conscience qui apparaît sous forme de fantômes portant les traces des violences passées (CE2-3), et qui lui déclarent que le conjoint violent ne changera pas (Hernández Orellana, Kunert, 2013). C'est grâce à cette prise de conscience que l'épouse, dont l'identité créée par le modèle mariano était une femme soumise et obéissante, n'a plus peur de regarder son agresseur droit dans les yeux (CE4). 


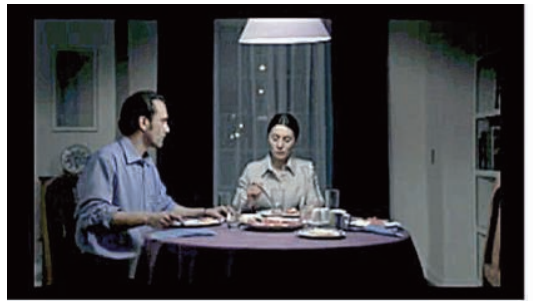

CE1

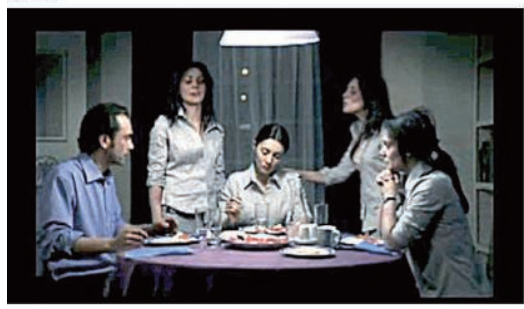

CE3

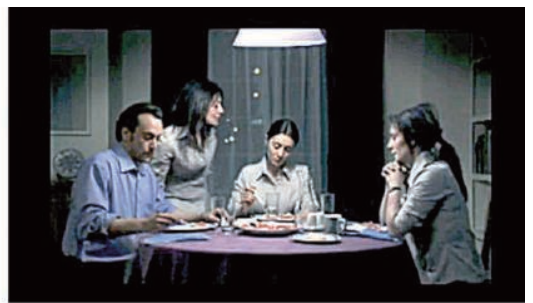

CE2

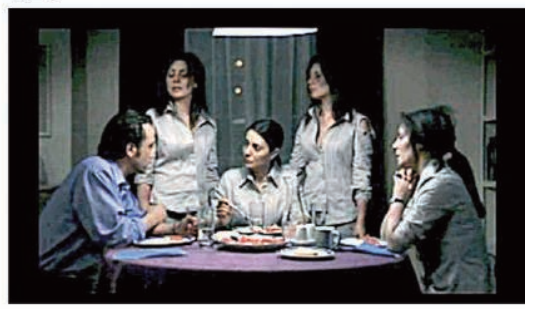

$\mathrm{CE}_{4}$

Ce nouveau type d'épouse (tout comme ses variantes : la compagne, la petite amie) fut mis en avant lors de la campagne de prévention de 2009, la dernière du gouvernement Bachelet. Ce tournant dans le changement d'identité des femmes est lié à l'évolution de la politique publique et plus généralement, aux conditions politiques. D'une part, le Sernam a décidé de construire les campagnes sous l'angle de la prévention et non plus de la sensibilisation, car d'après cet organisme, les Chiliens et les Chiliennes étaient déjà sensibilisés au sujet ${ }^{26}$. Si l'objectif restait celui de donner de la visibilité à la violence faite aux femmes, le Sernam a cherché cette fois à montrer un aspect plus positif des femmes et à donner de l'espoir aux victimes. D'autre part, la ministre avait une idée précise de ce que devait être l'évolution des campagnes sur cette thématique : ainsi la représentation d'une femme dotée d'empowerment ${ }^{27}$ est partie d'une construction de l'instrument de communication ${ }^{28}$. Enfin, le contexte politique a joué un rôle : les taux de popularité de Michelle Bachelet ${ }^{29}$ vers la fin de son mandat l'ont propulsée au rang de candidate la mieux placée pour l'élection présidentielle de $2013^{30}$. Étant donné que le Sernam a agi comme «le

26. Le Sernam ne dispose pas des outils permettant de mesurer la réception des campagnes de prévention. Ses estimations reposent sur les statistiques concernant le taux de plaintes déposées.

27. Nous utilisons ici la notion d'empowerment (empoderada), issue de l'approche féministe : "C'est un processus qui permet d'acquérir le sens d'une identité reposant sur la conscience de sa propre valeur et sur l'égalité [...] Tant que les femmes n'auront pas conscience qu'elles méritent des droits, elles ne seront pas empowered » (Kabeer cité par Bacqué et Biewener, 2013)

28. C'est ce qu'explique l'ancienne ministre du Sernam, Laura Albornoz, dans un entretien avec l'auteure de cet article réalisé à Santiago, le 2 mai 2011.

29. En octobre 2009 , la présidente fut plébiscitée par $78 \%$ de la population, d'après le sondage CEP (voir bibliographie).

30. Au Chili, pour solliciter sa réélection, le ou la présidente sortante doit laisser passer un mandat (4 ans). 
bras $»^{31}$ de la présidente, il n'est pas étonnant qu'il ait essayé de donner aux Chiliennes une image semblable à celle que Mme Bachelet, dans sa fonction, transmettait symboliquement : le dépassement de l'identité féminine, enfermée dans l'espace privé et construite sur la maternité, par «la (re)connaissance du désir de pouvoir politique et public» (Olea, 2010).

Tout cela a permis au Sernam de renverser le paradigme de la femme victime jusque-là mise en scène symboliquement par les campagnes de communication. En 2009, face à une caméra, différents types de femmes - censées représenter différents types de Chiliennes - s'expriment de façon affirmative en disant : «je m'aime telle que je suis, je décide où je vais, je n'ai pas peur, mes filles grandiront sans crainte, je m'habille comme je veux, mon travail a autant de valeur [que le tien], mon opinion a de l'importance. Parce que j'ai des droits, ne lève jamais la main sur moi ». Pour la première fois dans ces campagnes, les femmes ont investi l'espace public (la rue, le bureau, les transports en commun) pour s'exprimer et elles se sont adressées à un interlocuteur implicite (leur compagnon) à travers l'utilisation d'une phrase exhortative, qui clôt leur message. Dans le discours de l'État véhiculé par cette campagne, l'absence de l'homme agresseur - figure qui, jusqu'à la campagne précédente, était essentielle pour représenter les rapports de couple dans la famille patriarcale - est significative. À la différence des femmes représentées jusqu'en 2008 , celles de 2009 ne sont pas soumises à l'opinion de leur compagnon. Au contraire, elles montrent la confiance qu'elles ont en elles-mêmes par des phrases affirmatives qui construisent symboliquement un rapport de pouvoir plus égalitaire au sein du couple.

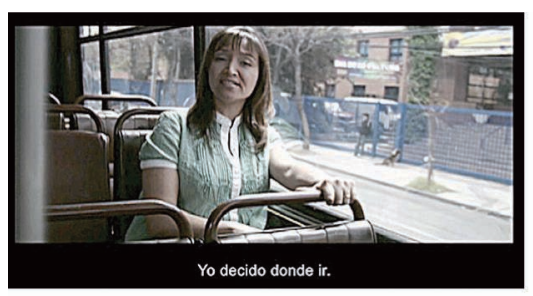

CE5 « Je décide où je vais»

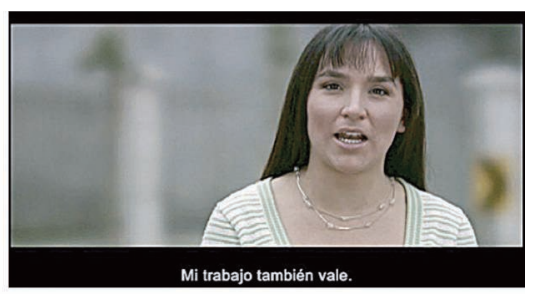

CE6 « Mon travail a autant de valeur que le tien »

Captures d'écran spot 2009 : «Entre un homme et une femme, maltraitance zéro »

31. Texte original : el brazo (ma traduction). Expression utilisée par Laura Albornoz dans l'entretien cité (note 28) 
Pour conclure, on peut observer que le gouvernement Bachelet voulait mettre en lumière la violence contre les femmes au Chili : à cette fin, il a largement utilisé l'instrument informatif et communicationnel. Fait important, dans les campagnes de la période étudiée, l'auteur des violences est clairement identifié (le mari ou compagnon de la victime). Ainsi, entre 2006 et 2008 , le discours institutionnel a construit un type de victime et, inversement, il a construit un type d'homme agresseur. Tout en favorisant la reconnaissance des violences faites aux femmes en tant que problème public, ces campagnes n'en ont pas moins assigné à celles-ci et aux hommes des identités caractéristiques du modèle familial patriarcal.

Mais progressivement, une nouvelle identité des Chiliennes s'est dessinée à travers ces campagnes de prévention. Cette construction est apparue au fur et à mesure que des rôles traditionnellement associés aux femmes, comme celui de mère et d'épouse, ont été marginalisés dans les dispositifs de communication. La campagne de 2009 marque une étape dans la construction de l'identité des femmes chiliennes : au-delà d'une rupture avec le paradigme antérieur caractéristique de la famille patriarcale, le Sernam a montré, plus largement, des femmes empoderadas comme la Présidente elle-même. Si l’identité des femmes victimes et des hommes agresseurs était construite à travers leurs différences, le gouvernement Bachelet essaye de construire symboliquement un nouveau modèle de famille, désormais composé par des individus égaux. Des études en réception de ces campagnes de prévention pourront peut-être nous éclairer sur la réussite de cette communication publique.

Lors du deuxième mandat de Mme Bachelet, le Sernam poursuit le recadrage de la politique publique envers les femmes initié dans son premier gouvernement. Depuis 2014, l'organisme travaille davantage sur l'individuation des femmes, y compris dans la thématique abordée dans cet article. Désormais, le Sernam s'attaque aux «violences contre les femmes » dans toute leur ampleur: dans le discours institutionnel, des actes tels que le harcèlement de rue et la violence économique font partie intégrante de ces violences.

\section{Références}

Araujo Kathya, Guzmán Virginia, Mauro Amalia, 2000, «El surgimiento de la violencia doméstica como problema público y objeto de políticas », Revista Cepal, nº70, avril, p. 133-145.

BACquÉ Marie-Hélène, BIEWEner Carole, 2013, L'empowerment, une pratique émancipatrice, Paris, La Découverte (Politique et sociétés).

Bereni Laure, Chauvin Sébastien, Jaunait Alexandre, Reveillard Anne, 2008, Introduction aux Gender Studies. Manuel des études sur le genre, Bruxelles, De Boeck Université (Ouvertures politiques). 
CEP, Encuestas CEP (Centro de Estudios Públicos), en ligne : http://www.cepchile.cl/ dms/archivo_4487_2631/encCEP_Octubre2009.pdf (consulté le 4 mars 2013).

Forstenzer Nicole, 2011, "L'institutionnalisation de la "perspective de genre" dans le Chili de la post-dictature. Enjeux et acteurs des va-et-vient de la politisation-

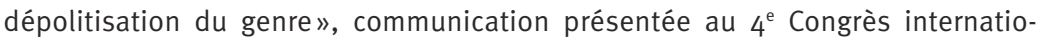
nal des associations francophones de sciences politiques, "Être gouverné au $21^{\mathrm{e}}$ siècle », Bruxelles, 20-22 avril 2011.

GofFMAN Erving, 2002, L'arrangement des sexes, Paris, La Dispute (Le genre du monde). Hernández Orellana Myriam, 2012, "La formule femicidio dans la presse chilienne. Un retour aux crimes passionnels? » Discours politiques en Amérique Latine. Représentations et imaginaires, M. Donot, M. Pordeus éd., Paris, L'Harmattan, p. 133-148.

- 2014, "Las campañas de comunicación contra la violencia intrafamiliar en el Chile de Michelle Bachelet. Rupturas y continuidades en la representación de las mujeres (víctimas) », Actes du $8^{\mathrm{e}}$ Congrès du GRIMH, Image et Genre. Masculin Féminin, Université Lumière Lyon 2, 15-17 novembre 2012, p. 209-222.

Hernández Orellana Myriam, Kunert Stéphanie, 2013, «Ethos de l'État et pathos communicationnel. Les campagnes gouvernementales de lutte contre les violences faites aux femmes (Chili, France, 2006-2010)», Semen, n³6, Les nouveaux discours publicitaires, p. 155-170.

Houdebine Anne-Marie, 1994, "Convergence / divergence / périphérie. Perspective dynamique et descriptive en sciences du langage : linguistique et sémiologie », Travaux de linguistique, $\mathrm{n}^{\circ}$ 5-6, Université d'Angers, p. 47-51.

- 2010, "De l'imaginaire linguistique à l'imaginaire culturel via la sémiologie des indices», Sémeion hors série, De l'imaginaire linguistique à l'imaginaire culturel, p.11-38.

JASPARD Maryse, 2011, Les violences contre les femmes, Paris, La Découverte (Repères).

LaRraín Soledad, Rodríguez Teresa, 1993, "Los orígenes y el control de la violence doméstica en contra la mujer», Género, mujer y salud en las Américas, E. Gómez éd.,Washington DC, OPS, publication scientifique $n^{\circ}$ 541, p. 202-209.

LASCOUmes Pierre, Le GalÈs Patrick éd., 2004, Gouverner pour les instruments, Paris, Presses de Sciences Po, 2004.

MARQUeS-PEREIRA Bérengère, 2011, "De la féminisation des démocraties au genre comme ressource politique. Le cas chilien », Cahiers du genre, hors-série, p.155-133.

MuLLER Pierre, 2006 [1990] Les politiques publiques, Paris, PUF (Que sais-je ?).

OLEA Raquel, 2010, «Michelle Bachelet. Fases y facetas de su representación pública », Yvotamos por ella. Michelle Bachelet : miradas feministas, A. Burotto, C. Torres éd., Santiago, Fundación Instituto de la Mujer, p. 17-34.

OLlivier-YAniv Caroline, 2013, «Communication, prévention et action publique : proposition d'un modèle intégratif et configurationnel. Le cas de la prévention du tabagisme passif», Communication et langages, juin, p. 93-111.

- 2006, "La communication publique. Communication d’intérêt général et exercice du pouvoir", Sciences de l'information et de la communication. Objets, savoirs, discipline, S. Olivesi éd., Grenoble, Presses universitaires de Grenoble, p. 97-112.

Oger Claire, Ollivier-Yaniv Caroline, 2003, «Analyse du discours institutionnel et 
sociologie compréhensive. Vers une anthropologie des discours institutionnels», Mots. Les langages du politique, $\mathrm{n}^{\circ} 71$, Mondialisation(S), p. 125-144.

Rıco Nieves, 1992, «Violencia doméstica contra la mujer en América Latina y el Caribe. Propuestas para la discusión », Santiago, Cepal (Unidad Mujery Desarrollo).

SoulAGES Jean-Claude, «Identités discursives et imaginaires figuratifs», L'imaginaire linguistique, A.-M. Houdebine éd., Paris, L’Harmattan, 2002, p. 103-109.

\section{Corpus}

SERnAm, 2008, "Bases técnicas para la contratación del diseño creativo de una campaña de prevención de la violencia contra la mujer, para este año 2008 », Santiago, Sernam, Departamento de Comunicaciones, $7 \mathrm{p}$.

- 2009, Documento de trabajo ${ }^{\circ}$ 121, Detección y análisis de la violencia intrafamiliar en la Región Metropolitana y La Araucania, 48 p.

- 2007, Agenda de Género 2006/2010, Gobierno Presidenta Michelle Bachelet Jeria, $80 \mathrm{p}$. 